\title{
Ovarian hormones influence eating disorder symptom variability during the menopause transition: A pilot study
}

\author{
Jessica H. Baker ${ }^{\mathrm{a},}$, Tory Eisenlohr-Moul ${ }^{\mathrm{b}}$, Ya-Ke Wu ${ }^{\mathrm{c}}$, Crystal E. Schiller ${ }^{\mathrm{a}}$, Cynthia M. Bulik ${ }^{\mathrm{a}, \mathrm{d}, \mathrm{e}}$, \\ Susan S. Girdler ${ }^{\mathrm{a}}$ \\ ${ }^{a}$ Department of Psychiatry, University of North Carolina at Chapel Hill, United States \\ ${ }^{\mathrm{b}}$ Department of Psychiatry, University of Illinois at Chicago, United States \\ ${ }^{c}$ School of Nursing, University of North Carolina at Chapel Hill, United States \\ ${ }^{\mathrm{d}}$ Deparmtent of Nutrition, University of North Carolina at Chapel Hill, United States \\ ${ }^{\mathrm{e}}$ Department of Medical Epidemiology and Biostatistics, Karolinska Institutet, Stockholm, Sweden
}

A R T I C L E I N F O

Keywords:

Ovarian steroid hormones

Estradiol

Progesterone

Eating disorders

\begin{abstract}
A B S T R A C T
Objective: Eating disorder symptoms change in a predictable pattern over the menstrual cycle such that changes in symptoms are triggered by changes in the ovarian hormones estradiol (E2) and progesterone (P4). To date, work in this area has focused exclusively on young adult women. The objective of this pilot study was to examine the effect of E2 and P4 on eating disorder symptom change in midlife women during early perimenopause. Method: Participants included women aged 42-52 in early perimenopause $(n=8)$. In-home self-assessments were completed for one menstrual cycle or 40-days, whichever occurred first. In-home self-assessments included collecting saliva samples each morning for E2 and P4 assays and completing online study questionnaires at the end of each day. Multilevel regression models examined the associations of E2 and P4 with daily symptoms of binge eating and body dissatisfaction.

Results: E2 was positively associated with binge eating when P4 was high, but not when P4 was low. E2 was inversely associated with body dissatisfaction when P4 was low, but positively associated with body dissatisfaction when P4 was high. However, the simple slopes for the effect of E2 at both high and low P4 were not significant for body dissatisfaction.

Conclusions: Despite the pilot nature of this study, results are broadly consistent with the young adult literature indicating that P4 levels shape the impact of E2 on eating disorder symptoms. Larger studies with the inclusion of key moderators to account for individual heterogeneity are needed to confirm and extend these findings.
\end{abstract}

\section{Introduction}

Eating disorder symptoms fluctuate across the menstrual cycle, and within-person change in estradiol (E2) and progesterone (P4) account for these phase differences (Baker, Girdler, \& Bulik, 2012). Previous work indicates that the mid-luteal phase of the menstrual cycle-when both E2 and P4 are higher-is when binge eating and emotional eating are highest (Edler, Lipson, \& Keel, 2007; Klump et al., 2013). There is also an interactive effect of changing levels of E2 and P4 on binge eating and emotional eating such that binge eating and emotional eating are exacerbated when both E2 and P4 are higher (Klump et al., 2013, 2014). Importantly, work in this area has failed to attend to important times of ovarian hormone change for women such as the menopause transition (i.e., perimenopause). Indeed, perimenopause has been hypothesized as a period of vulnerability for an eating disorder (Baker \& Runfola, 2016) as initial reports suggest the prevalence of eating disorders is increased in midlife women at perimenopause (Mangweth-Matzek et al., 2013).

Perimenopause is characterized by changes in ovarian hormone levels, namely erratic change in E2. At perimenopause, E2 dominance can occur such that while $\mathrm{P} 4$ levels remain relatively low and stable (when ovulation is not occurring), E2 levels can reach concentrations higher than those observed during pre-menopause (Santoro, Brown, Adel, \& Skurnick, 1996). Further, differing trajectories of E2 change have been observed during perimenopause with some women experiencing a slow E2 decline whereas others experience more frequent

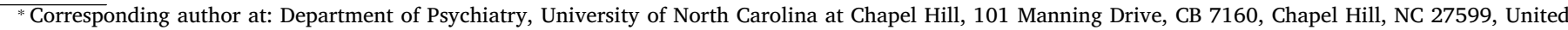
States.

E-mail address: jessica_baker@med.unc.du (J.H. Baker).
} 
highs and lows (Tepper et al., 2012). Taken together with the compelling evidence that changing E2 and P4 levels are involved in eating disorder symptom risk and fluctuation during young adulthood, this provides a strong rationale for exploring the role of ovarian hormones in eating disorder symptom fluctuation at perimenopause. Although the hormone changes that occur during young adulthood and at perimenopause are not identical, both represent periods of time when significant change in ovarian hormones occurs-which may ultimately influence eating disorder risk (Baker \& Runfola, 2016).

Thus, the aim of this pilot study was to preliminarily examine the interactive effect of changes in E2 and P4 on two core, transdiagnostic eating disorder symptoms: binge eating (BE: recurrent episodes of eating an unusually large amount of food in a short period time with an associated loss of control) and body dissatisfaction (BD: negative thoughts and feelings about one's body) in midlife women during perimenopause.

\subsection{Hypotheses}

Based on previous studies including young adult women, (Klump et al., 2013, 2014) we hypothesize a significant interaction will be observed between E2 and P4 such that higher levels of BE and BD will be observed when both E2 and P4 are high. Because of the pilot nature of this study, we focus only on the interactive effect of changing E2 and $\mathrm{P} 4$, the finding that is most consistently observed in previous work in non-clinical samples, and do not interpret main effects.

\section{Material and methods}

\subsection{Participants}

Eligible participants included women aged 42-52 in early perimenopause who reported a history of at least two loss of control eating episodes in the month prior to study enrollment $(n=8)$. For study enrollment purposes only, loss of control eating was defined as an episode of eating in which the participant felt as if they could not resist the urge to eat and/or could not stop eating. Menopause status was determined based on menstrual bleeding patterns during the past year using STRAW criteria +10, (Harlow et al., 2007) the gold standard defining menopause status. Early perimenopause was defined as having a menstrual period in the previous 3-months but experiencing at least a 7-day change in the length of the menstrual cycle between menstruation. Exclusion criteria included: surgical removal of ovaries and/or uterus; hormone supplementation or contraceptive use; steroid use in the previous 8-weeks; pregnancy or lactation in the past 6-months; or a genetic or medication condition known to influence ovarian hormone levels, weight, or appetite. This study was approved by the University of North Carolina IRB and participants provided written informed consent.

\subsection{Procedure}

Participants attended two study visits and completed in-home selfassessments. At Visit 1 participant eligibility was confirmed, baseline measures were completed, height and weight were measured, and data collection procedures were reviewed. In-home self-assessments included collecting saliva samples each morning for E2 and P4 assays and completing online study questionnaires at the end of each day. Selfassessments were completed for one menstrual cycle (day one of menses to day one of menses) or 40-days, whichever occurred first. For comparability purposes, procedures for saliva collection and completion of the self-report questionnaires were modelled after previous studies (Edler et al., 2007; Klump, Keel, Culbert, \& Edler, 2008, 2013). At Visit 2 participants returned the saliva samples and were debriefed.

\subsection{Measures}

\subsubsection{Estradiol and progesterone}

Saliva samples were collected daily by the participants in their home. Participants were instructed to collect $4 \mathrm{ml}$ of saliva (indicated by a line on the provided collection tubes) each morning within 30minutes of waking up and before brushing teeth, eating, drinking, or smoking. Participants stored the collection tubes in a provided cooler in their home freezer until returning for Visit 2.

\subsubsection{Daily eating disorder symptoms}

The Eating Pathology Symptom Inventory (EPSI; Forbush et al., 2013) examined baseline and daily eating disorder symptoms. At baseline, participants completed the full EPSI, which includes 8 subscales, to assess various eating disorder symptoms, with higher scores indicating more pathology. For the daily, evening assessment only the Binge Eating and Body Dissatisfaction subscales were included. For the daily assessment, the EPSI directions and question wording were modified, with permission, to reflect symptoms experienced for that day only (Forbush, personal communication). The EPSI was scored according to the scoring instructions (Forbush et al., 2013).

\subsubsection{Daily negative and positive affect}

The Positive and Negative Affect Scale (PANAS; Watson et al., 1988) was used to assess positive and negative affect.

\subsection{Statistical analyses}

\subsubsection{Data coding and preparation}

Analyses of within-person links between ovarian hormones and eating disorder symptoms were modeled after those used by Klump et al. (2013). In order to reduce impact of random environmental factors and hormone pulsatility, five-day rolling averages were calculated for hormones, $\mathrm{BE}, \mathrm{BD}$, and negative affect. Next, each of these rolling averages were converted to within-person $\mathrm{Z}$ scores based upon each participant's mean and standard deviation for that repeated measures variable across data collection. Use of these within-person standardized values is ideal for examining the association between hormones and eating disorder symptoms, because they index the extent to which changes in a woman's hormones, relative to her own typical levels and flux, predict changes away from her typical levels and flux in BE/BD (Klump et al., 2008). Coding of menstrual cycle phase was considered; however, given the erratic nature of observed hormone flux (which is expected and indicative of the menopause transition) in the majority of women, clear phases were not identifiable.

\subsubsection{Multilevel modeling}

Multilevel regression models tested study hypotheses. To account for serial correlation of daily repeated measures, an autoregressive (t-1) covariance structure was specified using a REPEATED statement in SAS PROC MIXED. Denominator degrees of freedom were determined using the Kenward-Roger method (Kenward \& Roger, 1997). Hypotheses were tested in two different models predicting daily $\mathrm{BE}$ or daily $\mathrm{BD}$ from daily E2, daily $\mathrm{P} 4$, and their interaction with both daily negative affect and Visit 1 body mass index (BMI) included as covariates.

\section{Results}

\subsection{Descriptive information}

Women reported a mean age of $45.9(\mathrm{SD}=5.0)$, mean BMI of 33.1 $(\mathrm{SD}=8.1)$, and a majority were Caucasian $(n=6)$, non-Hispanic $(n=7)$. Most women had a college education $(n=5)$ and were married or living with a partner $(n=7)$. At baseline, the mean BE score was $18.2(\mathrm{SD}=9.0)$ and the mean $\mathrm{BD}$ score was $20.3(\mathrm{SD}=5.0)$, which is slightly higher than young adult samples (Forbush et al., 
2013). The mean score for negative affect at baseline was 15.0 $(S D=5.4)$ and positive affect was $28.0(S D=6.0)$.

\subsection{Intraclass correlations of repeated outcomes}

Intraclass Correlations (ICCs) were calculated for unstandardized BE and BD. The ICC for BE was .59, indicating that $59 \%$ of the variance in $\mathrm{BE}$ was attributable to stable between-person differences; the ICC for $\mathrm{BD}$ was .84 , indicating that $84 \%$ of the variance in $\mathrm{BD}$ was attributable to stable between-person differences. Although the preponderance of the variance in these outcomes was attributable to trait-like differences in symptoms, there was a significant degree of within-person variance. In order to replicate prior studies in this area (e.g., Edler et al., 2007; Klump et al., 2013), we utilize person-standardized outcomes, which means that the daily outcomes contain only within-person variance, and therefore no between-person predictors were included (since betweenperson variance in outcomes had been removed).

\subsection{Effects of ovarian hormones on daily eating disorder symptoms}

Consistent with prior work, interactions of daily E2 and P4 were observed for both BE and BD (Table 1). Simple slopes of E2 on BE indicated that increasing E2 was strongly associated with higher BE when P4 was high (Fig. 1, top panel; $p=0.005$ ), but was not associated with $\mathrm{BE}$ when P4 was low (Fig. 1, top panel; $p=0.91$ ). Simple slopes of E2 on BD indicated that increasing E2 was also associated with higher BD when P4 was high (see Fig. 1, bottom panel; $p=0.17$ ), but increasing E2 was associated with lower BD when P4 was low (see Fig. 1, bottom panel; $p=0.17$ ). However, simple slopes for BD were not significant. Post-hoc power analyses indicated power was only adequate to detect large effect sizes; however, the number of observations in this pilot study was similar to those utilized in other samples (Edler et al., 2007; Klump et al., 2008). Results remained the same whether or not current BMI and daily negative effect were entered as between and withinperson covariates, respectively.

\section{Discussion}

Similar to young adults, within-person changes in ovarian hormones interacted to predict changes in BE and BD symptom scores for women at perimenopause. Our results are broadly consistent with the prior finding in younger women (Klump et al., 2013, 2014; Racine et al., 2012) that daily levels of P4 may shape the impact of daily E2 on eating

Table 1

Multilevel Models Predicting Daily Eating Disorder Symptoms from 5-Day Person-Standardized Rolling Averages of Ovarian Steroids.

\begin{tabular}{|c|c|c|c|c|}
\hline \multirow{3}{*}{ Parameter } & \multicolumn{4}{|c|}{ Daily Outcome } \\
\hline & \multicolumn{2}{|c|}{ Binge Eating } & \multicolumn{2}{|c|}{ Body Dissatisfaction } \\
\hline & Estimate & $S E$ & Estimate & $S E$ \\
\hline Intercept & -0.07 & 0.19 & -0.04 & 0.19 \\
\hline BMI & -0.02 & 0.19 & -0.09 & 0.21 \\
\hline Daily Negative Affect & 0.10 & 0.07 & -0.06 & 0.06 \\
\hline E2 & 0.15 & 0.08 & 0.01 & 0.08 \\
\hline P4 & $-0.20^{*}$ & 0.10 & -0.07 & 0.09 \\
\hline $\mathrm{E} 2 \times \mathrm{P} 4$ & $0.16^{*}$ & 0.06 & $0.13^{* *}$ & 0.05 \\
\hline \multicolumn{5}{|l|}{ Simple Slopes of E2 on Outcomes } \\
\hline Effect of E2 at Low P4 (-1 SD) & -0.01 & 0.10 & -0.12 & 0.09 \\
\hline Effect of E2 at High P4 (+1 SD) & $0.31 * *$ & 0.11 & 0.13 & 0.10 \\
\hline
\end{tabular}

Note: $\quad * p<0.05, \quad * * p<0.01, \quad * * * p<0.001 . \quad$ E2 $=$ Estradiol, P4 = Progesterone, BMI = Body Mass Index. Body mass index was samplestandardized. E2, P4, Negative Affect, Binge Eating, and Body Dissatisfaction represent 5-day rolling average levels that have been person-standardized (see text for details). Significant fixed effects are shown in bold.
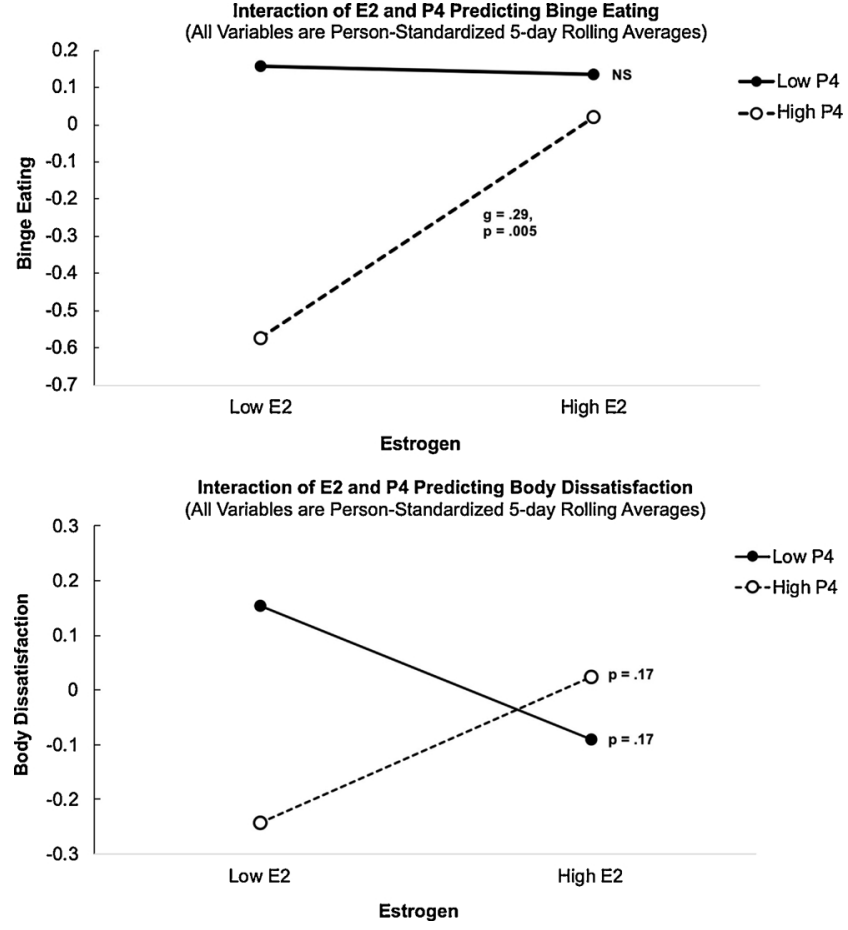

Fig. 1. Interactions of daily E2 and P4 in predicting daily binge eating (BE) and body dissatisfaction (BD) z-scores. Note. $* p<0.05, * * p<0.01$, $* * * p<0.001$. E2 = Estradiol, P4 = Progesterone. E2 and P4 represent 5-day rolling average levels that have been person-standardized in order to reflect within-person z-scores (see text for details). Significant fixed effects are shown in bold.

disorder symptoms yet how P4 shapes this impact may depend on the symptom examined. Specifically, we observed that within the context of low P4 (but not high P4), E2 was inversely associated with BD, but in the context of high P4, E2 was positively associated with BD and BE. Although simple slopes for BD were not significant, the follicular phase of the menstrual cycle is the phase associated with high E2 and low P4. The follicular phase follows menstruation, and menses may cyclically impact body image (Jappe \& Gardner, 2009). The cessation of menstruation and associated increases in E2 may thus more robustly impact BD. Taken together, cyclical ovarian hormone change may influence eating disorder symptom vulnerability in midlife women.

The findings of this pilot study align with preclinical work demonstrating a causal role of ovarian hormones on eating behavior. In ovariectomized animals, administration of only E2 suppresses appetite and eating behaviors via E2 receptors in the hypothalamus and the nucleus of the solitary tract in the brainstem, whereas administration of only P4 alone does not affect appetite (Butera, 2010). However, administration of P4 in the context of E2 appears to facilitate an increase in eating behaviors by reversing the appetite-suppressing effects of E2 (Kemnitz, Gibber, Lindsay, \& Eisele, 1989). Indeed, the preliminary findings in the present study are broadly consistent with these interactive effects of E2 and P4, since a protective effect of E2 on eating disorder symptoms was present only at low $\mathrm{P} 4$ that was reversed under conditions of high $\mathrm{P} 4$.

Because of the preliminary nature of this study, results must be interpreted with caution and limitations considered. First is the pilot sample size and thus associated concerns regarding power and generalizability. Because of the time intensive nature of the protocol, replicating the sophisticated protocol that has been implemented in young adults was favored at the expensive of sample size. Relatedly, as a pilot, we limit our interpretation of the results to the simple slopes given previous work in young adult samples has highlighted the importance of this interactive effect. Therefore, our a priori aim was to test 
the interactive effect of E2 and P4 on eating disorder symptoms in a midlife sample and compare findings to young adults. Second, given the erratic changes in E2 and P4 that characterize the perimenopause (as reflected in the samples collected here), we were unable to determine cycle phase. Third, we used a self-report questionnaire assessment of binge eating, which may not directly reflect DSM-defined binge eating. Finally, our sample only included women in early perimenopause. There may be a differential effect of ovarian hormones on eating disorder symptoms across the stages of menopause and post-menopause.

\section{Conclusions}

This is the first study to examine the influence of fluctuations in ovarian hormones on changes in eating disorder symptoms during a significant time of reproductive hormone flux for women. Similar to young adults, within-person change in ovarian hormones predicted changes in symptoms of $\mathrm{BE}$ and $\mathrm{BD}$ for women at perimenopause. Together, this suggests ovarian hormones may play a role in eating disorder etiology across the lifespan. Continuing to address the impact of ovarian hormones on eating disorder symptoms may lead to novel and personalized treatment approaches. For example, ovarian hormone stabilization or supplementation may be beneficial for some women with or at risk for an eating disorder-in particular during periods of significant reproductive hormone flux. Given perimenopause is defined by chaotic change in ovarian hormones, in particular E2, it may be a significant time of risk for eating disorder symptom onset, relapse, or exacerbation (Baker, et al., 2012).

Finally, experimental studies are needed to make causal interferences about the role of ovarian hormones in the risk for eating disorder symptoms and future studies need to include key moderators (e.g., negative life events) of response to ovarian hormone change. Perimenopause represents a quasi-experimental design to address the impact of changing ovarian hormones on behavior in humans. Continuing to address eating disorder symptoms during midlife and beyond is vital given women experience significant changes in ovarian hormones during this period of development.

\section{Contributors}

J.H.B. developed the study concept and design, collected the data, and led the drafting of the manuscript. Y.K.W. assisted drafting the manuscript under the supervision of J.H.B. T.E.M. performed the data analysis. C.E.S., C.M.B., and S.S.G. contributed to developing the study design. All authors contributed to the drafting of the manuscript. All authors approved the final version of the paper for submission.

\section{Acknowledgements}

This project was partially supported by the National Center for Advancing Translational Sciences, National Institutes of Health, through Grant Award Number UL1TR001111. Dr. Eisenlohr-Moul was funded by NIHR00109667 and Dr. Wu funded by National Institute of
Nursing Research, T32 NR007091. Funding sources had no role in the study design, collection, or analysis or interpretation of the data, writing the manuscript, or the decision to submit the paper for publication. Portions of the manuscript were presented at the 2018 International Conference on Eating Disorders, Chicago, IL.

\section{References}

Baker, J. H., Girdler, S. S., \& Bulik, C. M. (2012). The role of reproductive hormones in the development and maintenance of eating disorders. Expert Review of Obstetrics \& Gynecology, 7, 573-583. https://doi.org/10.1586/eog.12.54.

Baker, J. H., \& Runfola, C. D. (2016). Eating disorders in midlife women: A perimenopausal eating disorder? Maturitas, 85, 112-116. https://doi.org/10.1016/j.maturitas. 2015.12.017.

Butera, P. C. (2010). Estradiol and the control of food intake. Physiology \& Behavior, 99, 175-180.

Edler, C., Lipson, S. F., \& Keel, P. K. (2007). Ovarian hormones and binge eating in bulimia nervosa. Psychological Medicine, 37, 131-141.

Forbush, K. T., Wildes, J. E., Pollack, L. O., Dunbar, D., Luo, J., Patterson, K., \& Watson, D. (2013). Development and validation of the eating pathology symptoms inventory (EPSI). Psychological Assessment, 25, 859-878. https://doi.org/10.1037/a0032639.

Harlow, S. D., Crawford, S., Dennerstein, L., Burger, H. G., Mitchell, E. S., \& Sowers, M. F. (2007). Recommendations from a multi-study evaluation of proposed criteria for staging reproductive aging. Climacteric: the Journal of the International Menopause Society, 10, 112-119. https://doi.org/10.1080/13697130701258838.

Jappe, L. M., \& Gardner, R. M. (2009). Body-image perception and dissatisfaction throughout phases of the female menstrual cycle. Perceptual and Motor Skills, 108, 74-80.

Kemnitz, J. W., Gibber, J. R., Lindsay, K. A., \& Eisele, S. G. (1989). Effects of ovarian hormones on eating behaviors, body weight, and glucoregulation in rhesus monkeys. Hormones and Behavior, 23, 235-250.

Kenward, M. G., \& Roger, J. H. (1997). Small sample inference for fixed effects from restricted maximum likelihood. Biometrics, 53, 983-997.

Klump, K. L., Keel, P. K., Culbert, K. M., \& Edler, C. (2008). Ovarian hormones and binge eating: Exploring associations in community samples. Psychological Medicine, 38, 1749-1757. https://doi.org/10.1017/S0033291708002997.

Klump, K. L., Keel, P. K., Racine, S. E., Burt, S. A., Neale, M., Sisk, C. L., ... Hu, J. Y. (2013). The interactive effects of estrogen and progesterone on changes in emotional eating across the menstrual cycle. Journal of Abnormal Psychology, 122, 131-137. https://doi.org/10.1037/a0029524.

Klump, K. L., Racine, S. E., Hildebrandt, B., Burt, S. A., Neale, M., Sisk, C. L., \& Keel, P. K. (2014). Ovarian hormone influences on dysregulated eating: A comparison of associations in women with versus without binge episodes. Clinical Psychological Science: A Journal of the Association for Psychological Science, 2, 545-559. https://doi.org/10. $1177 / 2167702614521794$.

Mangweth-Matzek, B., Hoek, H. W., Rupp, C. I., Kemmler, G., Pope, H. G., Jr., \& Kinzl, J. (2013). The menopausal transition-a possible window of vulnerability for eating pathology. The International Journal of Eating Disorders, 46, 609-616. https://doi.org/ 10.1002/eat.22157.

Racine, S. E., Culbert, K. M., Keel, P. K., Sisk, C. L., Alexandra Burt, S., \& Klump, K. L. (2012). Differential associations between ovarian hormones and disordered eating symptoms across the menstrual cycle in women. The International Journal of Eating Disorders, 45, 333-344.

Santoro, N., Brown, J. R., Adel, T., \& Skurnick, J. H. (1996). Characterization of reproductive hormonal dynamics in the perimenopause. The Journal of Clinical Endocrinology and Metabolism, 81, 1495-1501. https://doi.org/10.1210/jcem.81.4 8636357.

Tepper, P. G., Randolph, J. F., Jr., McConnell, D. S., Crawford, S. L., El Khoudary, S. R., Joffe, H., \& Sutton-Tyrrell, K. (2012). Trajectory clustering of estradiol and folliclestimulatinghormone during the menopausal transition among women in the Study of Women'sHealth across the Nation (SWAN). The Journal of Clinical Endocrinology and Metabolism, 97, 2872-2880. https://doi.org/10.1210/jc.2012-1422.

Watson, D., Clark, L. A., \& Tellegen, A. (1988). Development and validation of brief measures of positive and negative affect: The PANAS scales. Journal of Personality and Social Psychology, 54, 1063-1070. 